\title{
Follow-up chest radiographic findings in patients with MERS-CoV after recovery
}

\section{Karuna M Das ${ }^{1,2}$, Edward Y Lee ${ }^{3}$, Rajvir Singh4, Mushira A Enani ${ }^{5}$, Khalid Al Dossari², Klaus Van Gorkom¹, Sven G Larsson², Ruth D Langer ${ }^{1}$}

${ }^{1}$ Department of Radiology, College of Medicine and Health Sciences, United Arab Emirates University, Al-Ain, UAE, ${ }^{2}$ Department of Medical Imaging, King Fahad Medical City, Riyadh, KSA, ${ }^{3}$ Department of Radiology and Medicine, Pulmonary Division, Boston Children's Hospital and Harvard Medical School, Boston, Massachusetts, USA, ${ }^{4}$ Department of Cardiology and Biostatistics, Hamad Medical Corporation, Doha, Qatar, 5 Department of Medicine (Infectious Disease), King Fahad Medical City, Riyadh, KSA

Correspondence: Dr. Karuna M Das, Department of Radiology, College of Medicine and Health Sciences, United Arab Emirates University, Al-Ain-17666, UAE. E-mail: daskmoy@gmail.com

\begin{abstract}
Purpose: To evaluate the follow-up chest radiographic findings in patients with Middle East respiratory syndrome coronavirus (MERS-CoV) who were discharged from the hospital following improved clinical symptoms. Materials and Methods: Thirty-six consecutive patients (9 men, 27 women; age range 21-73 years, mean \pm SD $42.5 \pm 14.5$ years) with confirmed MERS-CoV underwent follow-up chest radiographs after recovery from MERS-CoV. The 36 chest radiographs were obtained at 32 to 230 days with a median follow-up of 43 days. The reviewers systemically evaluated the follow-up chest radiographs from 36 patients for lung parenchymal, airway, pleural, hilar and mediastinal abnormalities. Lung parenchyma and airways were assessed for consolidation, ground-glass opacity (GGO), nodular opacity and reticular opacity (i.e., fibrosis). Follow-up chest radiographs were also evaluated for pleural thickening, pleural effusion, pneumothorax and lymphadenopathy. Patients were categorized into two groups: group 1 (no evidence of lung fibrosis) and group 2 (chest radiographic evidence of lung fibrosis) for comparative analysis. Patient demographics, length of ventilations days, number of intensive care unit (ICU) admission days, chest radiographic score, chest radiographic deterioration pattern (Types 1-4) and peak lactate dehydrogenase level were compared between the two groups using the student $t$-test, Mann-Whitney $U$ test and Fisher's exact test. Results: Follow-up chest radiographs were normal in 23 out of $36(64 \%)$ patients. Among the patients with abnormal chest radiographs $(13 / 36,36 \%)$, the following were found: lung fibrosis in $12(33 \%)$ patients GGO in $2(5.5 \%)$ patients, and pleural thickening in $2(5.5 \%)$ patients. Patients with lung fibrosis had significantly greater number of ICU admission days $(19 \pm 8.7$ days; $P$ value $=0.001)$, older age $(50.6 \pm 12.6$ years; $P$ value $=0.02)$, higher chest radiographic scores $[10(0-15.3) ; P$ value $=0.04]$ and higher peak lactate dehydrogenase levels $(315-370 \mathrm{U} / \mathrm{L} ; P$ value $=0.001)$ when compared to patients without lung fibrosis. Conclusion: Lung fibrosis may develop in a substantial number of patients who have recovered from Middle East respiratory syndrome coronavirus (MERS-CoV). Significantly greater number of ICU admission days, older age, higher chest radiographic scores, chest radiographic deterioration patterns and peak lactate dehydrogenase levels were noted in the patients with lung fibrosis on follow-up chest radiographs after recovery from MERS-CoV.
\end{abstract}

Key words: Lung fibrosis; MERS-CoV; Chest radiograph

\begin{tabular}{|l|l|}
\hline \multicolumn{2}{|c|}{ Access this article online } \\
\hline Quick Response Code: & \\
\cline { 1 - 2 } & Website: \\
\hline & www.ijri.org \\
\cline { 2 - 3 } & DOI: \\
&
\end{tabular}

This is an open access article distributed under the terms of the Creative Commons Attribution-NonCommercial-ShareAlike 3.0 License, which allows others to remix, tweak, and build upon the work non-commercially, as long as the author is credited and the new creations are licensed under the identical terms.

For reprints contact: reprints@medknow.com

Cite this article as: Das KM, Lee EY, Singh R, Enani MA, Dossari KA, Gorkom KV. et al. Follow-up chest radiographic findings in patients with MERS-CoV after recovery. Indian J Radiol Imaging 2017;27:342-9. 


\section{Introduction}

Outbreaks of infection of the Middle East respiratory syndrome coronavirus (MERS-CoV) are an emerging global health crisis. ${ }^{[1]}$ Until recently, most cases (1,223 with 520 deaths) have occurred in Saudi Arabia and exhibit a case fatality of approximately $43 \% .^{[2]}$ The morbidity and mortality caused by MERS-CoV are particularly alarming. The majority of affected patients typically present with a severe respiratory illness that requires hospitalization; the mortality rate approaches $60 \% .^{[3]}$

Chest radiography is found to be useful in the diagnosis of MERS-CoV by demonstrating lung abnormalities and in the evaluation of the progress of disease and its response to treatment. ${ }^{[4]}$ Two recently published studies reported MERS-CoV chest radiographic findings and showed that sequential chest radiographs were valuable in the evaluation of the early diagnosis and monitoring of the radiographic progression of the disease. ${ }^{[5-7]}$ Approximately $55 \%$ of MERS-CoV patients require ICU admission for respiratory failure and adult respiratory distress syndrome (ARDS). ${ }^{[5-7]}$ Survivors of ARDS have a clinically significant reduction in health-related quality of life and varied radiological changes. ${ }^{[8-10]}$ In the present study, we focused on investigating the lung parenchymal, airway, pleural, hilar and mediastinal abnormalities which can be present on short- and medium-term follow-up chest radiographs during the recovery period; this may have important clinical implications for understanding the sequelae of the disease and contribute to the future management of affected patients.

Therefore, the purpose of this study is to evaluate the follow-up chest radiographic findings in MERS-CoV patients who were discharged from the hospital following improved clinical symptoms.

\section{Materials and Methods}

\section{Participants}

This retrospective study was approved by the institutional review board with a waiver of informed consent. Our study ran from August 10, 2014 to September 02, 2015 and included 36 patients who were discharged from the hospital following recovery from the MERS-CoV infection. The final study population consisted of nine male and 27 female patients. Patient age ranged from 21 years to 73 years (mean \pm SD, $42.5 \pm 14.5$ years).

In our study population, MERS-CoV diagnosis was based on the World Health Organization (WHO) criteria. ${ }^{[1]}$ At our institution, the treatment protocol for non-ICU patients suspected of having MERS-CoV was empiric treatment with oseltamivir (Tamiflu) until screening for respiratory viruses excluded influenza $A$ and $H 1 N 1$. In addition, antibiotics (ceftriaxone and azithromycin) were started to manage the potential of bacterial pneumonia. For ICU patients, the same patient management policy was followed, but the choice of antibiotics was broader, such as piperacillin-tazobactam (Zosyn) with or without vancomycin (Vancocin). All 36 patients were discharged from the hospital after the following: 1) complete recovery from their current illness, 2) complete resolution of respiratory symptoms and oxygen dependency, 3) improved radiological parameters based upon serial radiographs and 4) improved laboratory parameters. All these patients fulfilled above mentioned criterion before discharge from the hospital.

The chest radiographic findings and computed tomographic findings of 36 out of 55 patients obtained during the active phase of the infection that are included in this report have previously been published. ${ }^{[5-7]}$ The current study focused on the retrospective analysis of follow-up chest radiographic findings of the 36 patients after they had recovered from MERS-CoV as this had not been previously investigated.

\section{Imaging technique}

Digital radiography equipment (Mobilett Plus; Siemens, Erlangen, Germany) was used to obtain anteroposterior (AP) projection radiographs with standardized parameters $[65 \mathrm{kV}$, 4-8 mAs according to body mass index (BMI) and focus-film distance of $100 \mathrm{~cm}$. Non-portable radiography equipment (Philips Medical Systems, Hamburg, Germany) was used to obtain anteroposterior (PA) and lateral projection radiographs with standard techniques at a 180-cm focus-film distance.

Among the 36 follow-up chest radiographs from the 36 patients, 31 (86\%) were obtained with a portable one-view technique (AP projection radiographs), while the remaining five $(14 \%)$ radiographs were obtained with standard chest two-view techniques (PA and lateral projection radiographs).

\section{Chest radiograph review}

Two thoracic radiologists with 20 (K.M.D) and 8 (K.D.) years of experience of interpreting chest radiographs independently reviewed all chest radiographs which had been randomized prior to the evaluation process. The reviewers reached a decision on the final interpretation by consensus. Although the two reviewers were aware of the MERS-CoV infection diagnosis in our study population, other information, such as clinical information, laboratory results, prior imaging or non-imaging studies, original radiologic interpretation of chest radiographs and the group into which each of the patients were categorized, was blinded to decrease potential reviewer bias. For the studies with interpretation discrepancies from the two initial reviewers, a third reviewer (S.G.L., with 40 years 
of experience) served as the arbitrator and made the final decision after independently reviewing the chest radiographs.

A dedicated radiology picture archiving and communication system (PACS) workstation (Centricity 2.1.2.1; GE Healthcare, Milwaukee, WI, USA) that is available in our department was used for the evaluation of the chest radiographs from our study population. During the evaluation, reviewers could manually alter the degree of radiodensity settings and zoom into areas of interest to achieve a complete and detailed assessment.

\section{Chest radiograph evaluation}

Initially, all chest radiographs were categorized into two groups, normal or abnormal, based on lung parenchymal, airway, pleural, hilar and mediastinal findings on follow-up chest radiographs. Abnormal follow-up chest radiographs were subsequently assessed by established criteria as detailed in the following sections.

Lung parenchyma and airways were evaluated for the following: 1) consolidation, 2) ground-glass opacity (GGO), 3) nodular opacity, and 4) reticular opacity (i.e., fibrosis). ${ }^{[12-18]}$ A diagnosis of consolidation was made when there was an area of increased opacity present that obscured the vessel margin and airway walls, with or without an air bronchogram. ${ }^{[14]} \mathrm{GGO}$ was defined as an area of increased lung opacity within which margins of pulmonary vessels may be indistinct. ${ }^{[14]} \mathrm{A}$ diagnosis of nodular opacity was considered when an opacity was focal and round in shape. Reticular opacity which signals fibrosis, was defined as linear opacities forming a mesh-like pattern. ${ }^{[12-18]}$ Follow-up chest radiographs were also evaluated for the presence of pleural thickening, effusion, or pneumothorax. Additionally, the hilar and mediastinum were assessed for lymphadenopathy. A hilar lymphadenopathy was considered to be present when there was an increase in the size and opacity of the hilum with a lobulated hilar contour and obscuration of the interlobular artery. ${ }^{[18,19]}$ Mediastinal lymphadenopathy was defined as the presence of the widening of one or more mediastinal contours at known anatomic lymph node sites. ${ }^{[19]}$

Furthermore, if any other thoracic abnormalities not included in the aforementioned categories during the evaluation process were detected on follow-up chest radiographs, the reviewers were instructed to record them.

Comparison of chest radiograph findings and clinical parameters

Patient demographics, length of ventilations days, number of ICU admission days, chest radiographic score, chest radiographic deterioration pattern (Types $1-4)^{[5-7]}$ and peak lactate dehydrogenase level ${ }^{[20,21]}$ were compared between the cohort's two study groups ${ }^{[5-7]}$ in order to evaluate the association with the presence of lung fibrosis seen on follow-up chest radiographs.

The chest radiographic score of MERS-CoV infection can be obtained by first dividing each lung into three zones and evaluating the levels of involvement in each zone. ${ }^{[2]}$ The development of MERS-CoV lesions within each lung zone is then scored as follows: $0=$ normal; $4=$ complete involvement of one zone; or, 24 = complete involvement of all six zones. ${ }^{[5]}$ The scores for all six zones per chest radiograph are added together to yield a cumulative chest radiographic score ranging from 0 to 24 , depending upon the involvement of the lung parenchyma.

Chest radiographic deterioration during disease progression can be classified into four types. ${ }^{[1-4]}$ Type 1 disease progression represents initial radiographic deterioration followed by improvement. ${ }^{[12,13]}$ Type 2 disease progression is defined as static radiographic changes with no discernible radiographic peak or a change in overall mean lung involvement of less than $25 \%$. Type 3 disease progression is represented by fluctuating radiographic changes with at least two radiographic peaks separated by a period of mild remission (remission is defined as a level of mean lung parenchyma involvement that differs from the peak level by $>25 \%$ ). Type 4 progression is defined as progressive radiographic deterioration.

\section{Statistical analysis}

Descriptive statistics in the form of mean, standard deviation, median and range as appropriate for age, length of ventilations days, number of ICU admission days, chest radiographic score, chest radiographic deterioration pattern, peak lactate dehydrogenase (LDH) level and frequency, with percentages for categorical variables, were calculated separately for the group of patients with lung fibrosis and without. The student $t$-test for normally distributed data and the Mann-Whitney $U$-test for non-normal distributed data were applied to assess statistically significant differences between with and without evidence of lung fibrosis groups. Fisher's exact tests were used to evaluate associations for categorical variables. A $P$ value 0.05 (two-tailed) was considered for the statistically significant level. The IBM SPSS version 21.0 (IBM, Armonk, NY, USA) statistical package was used for the analysis of the study.

\section{Results}

\section{Study cohort}

There were no significant gender differences between the two groups ( $p=0.41)$. However, group 2 patients with evidence of lung fibrosis were significantly older $(p=0.02)$ than group 1 patients and had a significantly greater number of ICU admission days ( $p=0.001)$. The mean $\pm \mathrm{SD}$, median, and range of follow-up of the 36 chest radiographs were $64.8 \pm 85$ days, 43 days, and 32-230 days, respectively. The 
mean \pm SD, median and range of lung fibrosis development of 12 patients were $82.4 \pm 66$ days 44 days, and 32-230 days, respectively.

\section{Chest radiograph findings}

Two reviewers (K.M.D., K.D.) were able to reach a consensus on all abnormal findings detected on follow-up chest radiographs. The follow-up chest radiographs were normal in 23 of $36(64 \%)$ patients. Abnormal chest radiographic findings were detected in 13 of $36(36 \%)$ patients and are shown in Tables 1, 2, and 3.

The most frequent abnormality found on follow-up chest radiographs in MERS-CoV patients who recovered was lung fibrosis which was seen in 12 of 36 (33\%) patients. Lung fibrosis was confined to one lobe in the 10 patients, including the right upper lobe in three $(8 \%)$ patients,

Table 1: Distribution of radiological findings noted in 13/36 patients with abnormal chest radiographs

\begin{tabular}{llc}
\hline Findings & Category & No $(\%)$ \\
\hline Lung fibrosis & & $12(33 \%)$ \\
Multiple & & $2(5.5 \%)$ \\
Solitary & Right lower & $3(8 \%)$ \\
& Left lower & $3(8 \%)$ \\
& Left mid & $2(5.5 \%)$ \\
& Right mid & $2(5.5 \%)$ \\
Pleural thickening & Left lower lobe & $1(2.8 \%)$ \\
Ground-glass opacities & Right lower lobe & $1(2.8 \%)$ \\
& Left lower lobe & $1(2.8 \%)$ \\
& Lingula & $1(2.8 \%)$ \\
\hline
\end{tabular}

left lower lobe in three $(8 \%)$ patients, right middle lobe in two (5.5\%) patients and lingula in two $(5.5 \%)$ patients [Figure 1]. The remaining lung fibrosis involved multiple zones in two (5.5\%) patients [Figures 2 and 3]. GGO was the next most frequently detected abnormality on follow-up chest radiographs as seen in two (5.5\%) patients and it was located in the lingula [Figure 4] in one patient and in the left lower lobe in one patient, respectively. No consolidation or nodular opacity was detected on follow-up chest radiographs.

In regard to pleural abnormality detected on follow-up chest radiographs in our study population, pleural thickening was seen in two patients and it was located in the right lower and left lower lobes, respectively [Figure 5]. No pleural effusion or pneumothorax was identified.

None of the patients had hilar and/or mediastinal lymphadenopathy. No other additional thoracic abnormalities were observed on follow-up chest radiographs in our study population.

\section{Comparison of chest radiograph findings and clinical parameters}

With regard to the extent of lung parenchymal involvement (chest radiographic score) during the course of the disease, the patients with evidence of lung fibrosis were associated with a significantly higher number of chest radiographic score $(P=0.04)$ [Table 2].

The association of lung fibrosis was highest (67\%) in the Type 2 radiographic deterioration pattern, followed by the

Table 2: Distribution of different clinical parameters with and without lung fibrosis in 36 Patients of MERS-CoV

\begin{tabular}{|c|c|c|c|}
\hline Characteristics & $\begin{array}{l}\text { Patients without lung fibrosis (Group 1) } \\
\qquad n=24\end{array}$ & $\begin{array}{l}\text { Patients with lung fibrosis (Group 2) } \\
\qquad n=12\end{array}$ & $P$ \\
\hline Mean age (years) & $39 \pm 14$ & $50.6 \pm 12.6$ & 0.02 \\
\hline Male/female ratio & $7 / 17(29.2 \%)$ & $2 / 10(16.7 \%)$ & 0.41 \\
\hline Average ventilation days & $3.8 \pm 7$ & $10 \pm 11.5$ & 0.09 \\
\hline ICU admission days & $9 \pm 7$ & $19 \pm 8.7$ & 0.001 \\
\hline Number of Abnormal Segment in Chest Radiograph* & $0.8(0-5)$ & $0.8(0-3.3)$ & 0.87 \\
\hline Chest radiographic score at the peak* & $1.05(0-14.1)$ & $10(0-15.3)$ & 0.04 \\
\hline Peak lactate dehydrogenase (LDH) $(\mathrm{U} / \mathrm{L})^{*}$ & $0(0-310)$ & $315(0-370)$ & 0.001 \\
\hline \multicolumn{4}{|l|}{${ }^{*}$ Figures are median (range) } \\
\hline Characteristics & $\begin{array}{l}\text { Patients without Fibrosis (Group 1) } \\
\qquad n=24\end{array}$ & $\begin{array}{l}\text { Patients with Fibrosis (Group 2) } \\
\qquad n=12\end{array}$ & $P$ \\
\hline Age & $39 \pm 14$ & $50.6 \pm 12.6$ & 0.02 \\
\hline Gender: Male/Female & $7 / 17(29.2 \%)$ & $2 / 10(16.7 \%)$ & 0.41 \\
\hline \multicolumn{4}{|l|}{ Radiographic deterioration pattern (Type 1-Type 4) } \\
\hline Type 1 & $4(16.7 \%)$ & $0(0 \%)$ & 0.13 \\
\hline Type 2 & $10(41.7 \%)$ & $8(66.7 \%)$ & 0.23 \\
\hline Type 3 & $0(0 \%)$ & $3(25 \%)$ & 0.01 \\
\hline Type 4 & $2(8.7 \%)$ & $0(0 \%)$ & 0.31 \\
\hline
\end{tabular}




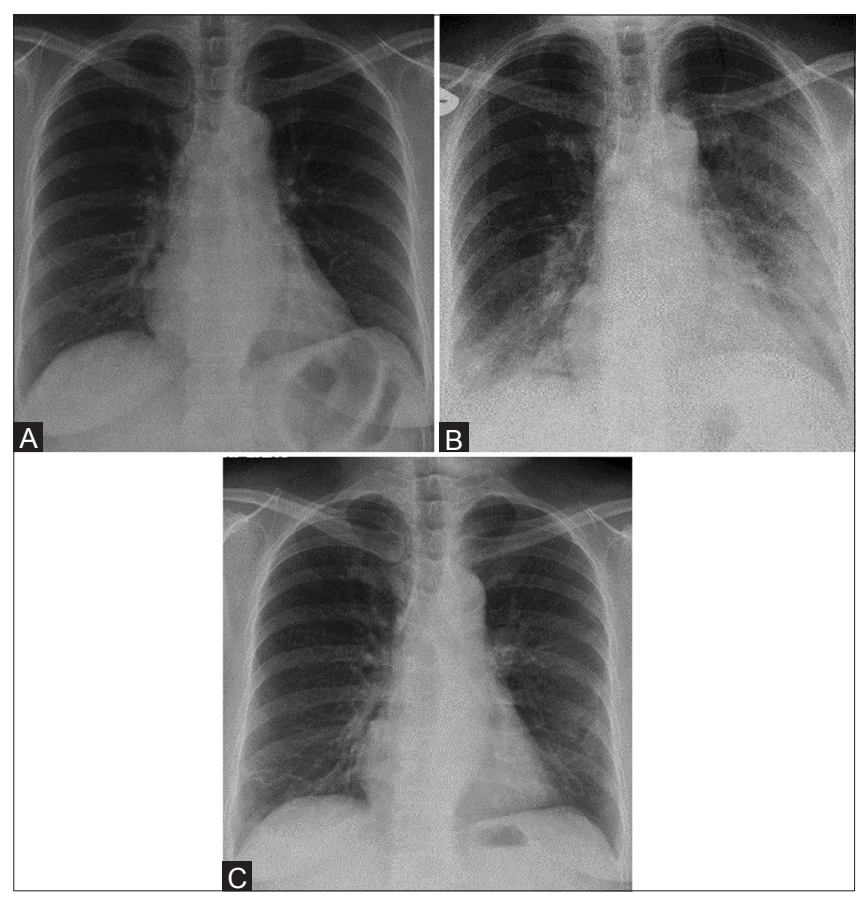

Figure 1 (A-C): A 58-year-old male with Middle East respiratory syndrome coronavirus (MERS-CoV), serial radiographs showing irregular reticular lines of fibrosis (A) Frontal chest radiograph obtained on two days before illness shows a normal chest radiograph. (B) A follow-up frontal chest radiograph obtained at day 5, shows ground-glass opacities in the right lower zone and left mid and lower zones. (C) A follow-up frontal chest radiograph obtained at day 33 shows unilateral multiple irregular reticular lines of fibrosis in the right lower and left mid zones

Type $3(25 \%, P=0.01)$ radiographic deterioration pattern [Table 3]. The association with the Type 4 radiographic deterioration pattern could not be ascertained due to lack of follow-up following the highest number of deaths in this group [Table 3].

The peak lactate dehydrogenase level was also higher in group 2 than in group 1 [315 (0-370 U/L) vs. $0(0-310 \mathrm{U} / \mathrm{L})]$ [Table 2]. The normal range of lactate dehydrogenase level at our institution is $121-214 \mathrm{U} / \mathrm{L}$.

\section{Discussion}

The results of our study show that a substantial portion (33\%) of the follow-up chest radiographs obtained from the MERS-CoV patients who recovered is abnormal. In our study population, the chest radiographic abnormalities are characterized by the presence of lung fibrosis, GGOs, and pleural thickening.

We believe that our finding of potentially permanent damage, such as lung fibrosis and pleural thickening, seen in a substantial portion of our MERS-CoV patients who recovered is important because typical viral pneumonia usually results in a complete recovery without any significant radiological consequences. ${ }^{[23]}$ The results

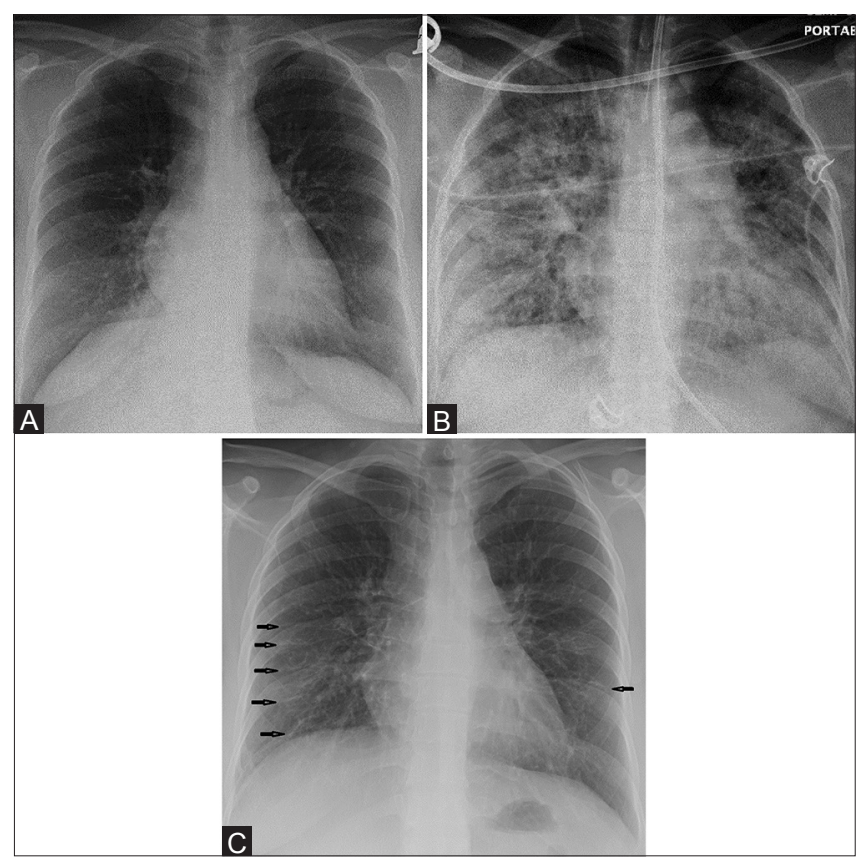

Figure 2 (A-C): A 33-year-old female with Middle East respiratory syndrome coronavirus (MERS-CoV), serial radiographs showing multiple irregular reticular lines of fibrosis on follow-up chest radiographs. The rest of the lung is completely free of any irregular reticular lines of fibrosis (A) Frontal chest radiograph obtained on the initial presentation shows the area of ground-glass opacity at the right cardio-phrenic angle. (B) A follow-up frontal chest radiograph obtained at day 20 , shows bilateral diffuse ground-glass opacities with occasional airspace consolidations. (C) A follow-up frontal chest radiograph obtained at day 230 , shows bilateral multiple irregular reticular lines of fibrosis (arrows)

of our study suggest that MERS-CoV may be a novel, more aggressive type of lung infection than other viral lung infections and that it may be associated with more significant morbidity in affected patients. In fact, our study also shows that lung fibrosis developed rather rapidly in the study population with a mean $\pm \mathrm{SD}$, median and range of lung fibrosis development of $82.4 \pm 66$ days 44 days, and $32-230$ days, respectively, after discharge from the hospital.

Lung fibrosis has been detected on follow-up imaging studies in patients who recovered from relatively new pulmonary infections such as swine influenza (i.e. H1N1) pneumonia ${ }^{[24]}$ and severe acute respiratory syndrome (SARS). ${ }^{[25]}$ We found that the incidence (33\%) of lung fibrosis seen in our study population is higher than the rate $(10 \%)$ of lung fibrosis seen in patients who recovered from swine influenza pneumonia. ${ }^{[24]}$ However, the incidence (33\%) of lung fibrosis seen in patients who recovered from MERS-CoV is similar to the incidence (38\%) of lung fibrosis detected in patients who recovered from SARS. ${ }^{[25]}$ Given that the presence of pulmonary fibrosis was evaluated based on follow-up chest radiographs in our study population, in comparison to the chest computed tomography (CT) used in another study that focused on detecting lung fibrosis in patients who recovered from SARS, we believe that the presence 


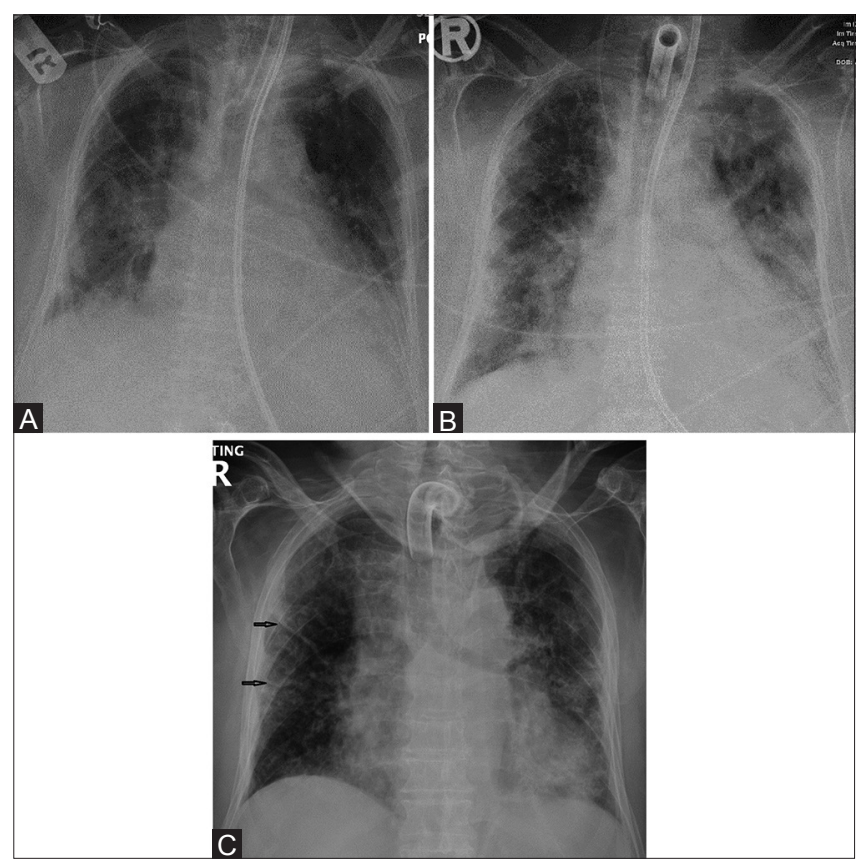

Figure 3 (A-C): A 73-year-old female with Middle East respiratory syndrome coronavirus (MERS-CoV), serial radiographs showing multiple thick reticular lines of fibrosis and sub-pleural reticular opacities on follow-up chest radiographs. (A) A frontal chest radiograph obtained on day 3 , of the initial presentation, shows bilateral ill-defined ground-glass opacities with air space disease at both lung bases. (B) A follow-up frontal chest radiograph obtained at day 19 , shows bilateral sub-pleural ground-glass opacities with occasional air space consolidations. (C) A follow-up frontal chest radiograph obtained at day 130 , shows unilateral thick multiple linear fibrotic parenchymal bands (arrows) in the right side along with sub-pleural reticulations, pleural thickening, and bilateral ground-glass opacities

of lung fibrosis would be much higher if lung fibrosis was evaluated on chest $\mathrm{CT}$ rather than on chest radiographs as in our study population. Unfortunately, most of our patients underwent chest radiographs rather than chest CT for a follow-up evaluation after MERS-CoV recovery, which prevented us from investigating pulmonary fibrosis with chest CT. A future study using chest CT for the detection of lung abnormalities in our study population would be helpful to gain a more precise evaluation as $\mathrm{CT}$ is more sensitive than chest radiographs for accurately detecting such abnormalities.

In our study, direct comparison of the follow-up chest radiographic findings with clinical data revealed several significant differences between patients with follow-up chest radiographic evidence of lung fibrosis and those without evidence of lung fibrosis. We found that patients with lung fibrosis detected on follow-up chest radiographs were associated with a greater number of ICU admission days, older age, higher chest radiographic score and higher peak lactate dehydrogenase level. In other words, we believe that these are the underlying risk factors and/or predictors for developing lung fibrosis in MERS-CoV patients who recovered. It is understandable that lung fibrosis develops
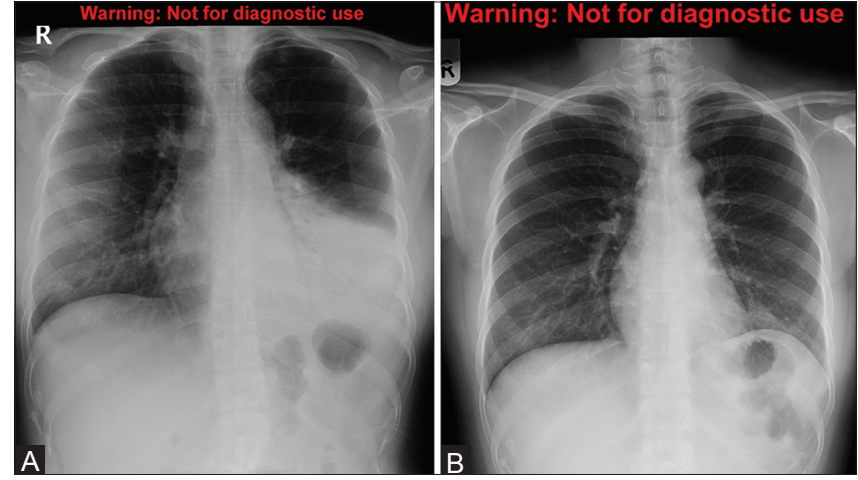

Figure 4 (A and B): A 24-year-old female with Middle East respiratory syndrome coronavirus (MERS-CoV), serial radiographs showing ground-glass opacities on follow-up chest radiographs. (A) A frontal chest radiograph obtained on the second day of the presentation shows the area of air space consolidation involving the lingual and left lower lobe. (B) Follow-up frontal chest radiograph obtained at 210 days shows only a small area of ground-glass opacity obscuring the left cardiac border

in patients who have been more severely infected with MERS-CoV (i.e. higher chest radiographic score) and who experience a longer infection period (i.e., greater number of ICU admission days). In addition, older patients with already aged lungs and decreased immune systems may not be able to completely recover from MERS-CoV and may be prone to develop viral-induced lung fibrosis. ${ }^{[26]}$

The exact triggers which initiate the lung fibrotic process remain unknown. Infectious agents, including both viruses and bacteria, have the capacity to cause alveolar epithelial cell injury and apoptosis. ${ }^{[24,27,28]}$ Presumably, because of the similar reaction, a considerable number of the patients in our cohort with MERS-CoV have developed pulmonary fibrosis. If infection plays a causal role in the process of pulmonary fibrosis, then it is possible that therapeutic strategies, utilizing currently available antiviral or antibiotic drugs, may be effective in modifying the course of this dreadful condition. ${ }^{[27,28]}$

In regard to our finding of the association between the presence of lung fibrosis on follow-up chest radiographs and higher peak lactate dehydrogenase level, we believe that this can be explained because the higher peak of lactate dehydrogenase level may indicate underlying lung tissue destruction (i.e. lung fibrosis). This finding of the higher peak of lactate dehydrogenase level has been seen in patients affected with SARS and shown to be a good independent predictor of a worse clinical outcome. ${ }^{[20,21]}$ Future studies focusing on understanding the underlying pathophysiology of developing lung fibrosis in this patient population would be valuable to better elucidate the direct underlying risk factors and their consequences.

This study has some limitations. First, some of the patients in our study had only frontal radiographs. Although we 


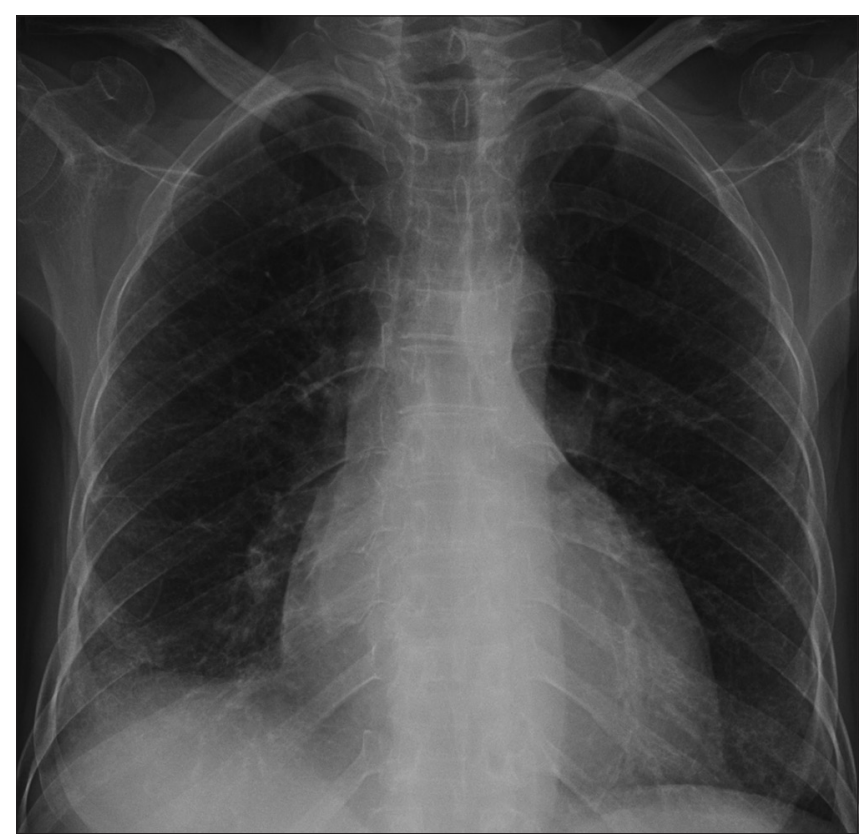

Figure 5: A 52-year-old female with Middle East respiratory syndrome coronavirus (MERS-CoV). A frontal chest radiograph obtained at day 168 , shows bilateral multiple irregular reticular lines of fibrosis along with obscured lateral aspect of the right hemidiaphragm and costo-phrenic angle in the same side due to pleural thickening

believe that this did not have a significant effect on our findings, we acknowledge that there may have been a limitation regarding the complete assessment of subtle retrocardiac abnormalities and small pleural effusions that can be better seen on lateral radiographs. However, we would like to emphasize that the reason for obtaining a single view (i.e. frontal radiograph only) was primarily due to our concerted effort to reduce the radiation exposure of our patient population. Second, as we have briefly mentioned in the discussion section, CT has better sensitivity than chest radiography in the diagnosis of lung fibrosis. Thus, the frequency of imaging findings such as lung fibrosis may have been underestimated in our study. A future study focusing on follow-up CT findings in patients who have recovered from MERS-CoV would be valuable in this regard. Lastly, our study focused on short- and intermediate-term follow-up imaging findings in our study population. A future study focusing on a long-term follow-up would be helpful for understanding the full spectrum of the sequelae of this new lung infection.

In conclusion, it is alarming that a substantial portion of the follow-up chest radiographs from the MERS-CoV patients who recovered are abnormal and characterized by potentially permanent lung damage such as lung fibrosis. Based on the observations made in our study, we believe that the greater number of higher ICU admission days, older age, higher chest radiographic score and higher peak lactate dehydrogenase level may have value in the prediction of lung fibrosis development in patients who have recovered from
MERS-CoV. However, we wish to stress that our findings are based on short- and intermediate-term follow-up evaluation. Future studies consisting of a larger patient population with a longer follow-up period would be necessary in order to confirm our findings and better determine the long-term outcomes of patients who recovered from MERS-CoV.

Financial support and sponsorship

Nil.

\section{Conflicts of interest}

There are no conflicts of interest.

\section{References}

1. Zaki AM, Van Boheemen S, Bestebroer TM, Osterhaus AD, Fouchier RA. Isolation of a novel coronavirus from a man with pneumonia in Saudi Arabia. N Engl J Med 2012;367:1814-20.

2. MERS-an uncertain future, Editorial review. Lancet Infect Dis 2015; 15:1115.

3. World Health Organization. Global Alert and Response (GAR). Corona virus infections. http://www.who.int/csr/disease/corona virus_infections/en/.

4. World Health Organization. Preliminary clinical description of severe acute respiratory syndrome. www.who.int/csr/sars/clinical/ en/. [Accessed 2003March 21].

5. Das KM, Lee EY, Jawder SE, Enani MA, Singh R, Skakni L, et al. Acute Middle East Respiratory Syndrome Coronavirus: Temporal Lung Changes Observed on the Chest Radiographs of 55 Patients. AJR Am J Roentgenol 2015;205:W267-74.

6. Das KM, Lee EY, Enani MA, Jawder SE, Enani MA, Singh R, Skakni L, et al. CT correlation with outcomes in 15 patients with acute Middle East respiratory syndrome corona virus. AJR Am J Roentgenol 2015;204:736-42.

7. Das KM, Lee EY, Langer RD, Larsson SG. Middle East Respiratory Syndrome Coronavirus: What Does a Radiologist Need to Know? AJR Am J Roentgenol 2015;206:1193-01.

8. Davidson TA, Caldwell ES, Curtis JR, Hudson LD, Steinberg KP. Reduced quality of life in survivors of acute respiratory distress syndrome compared with critically ill control patients. JAMA 1999;281:354-60.

9. Howling SJ, Evans TW, Hansell DM. The significance of bronchial dilatation on CT in patients with adult respiratory distress syndrome. Clin Radiol 1998;53:105-9.

10. Zapol WM, Trelstad RL, Coffey JW, Tsai I, Salvador RA. Pulmonary fibrosis in severe acute respiratory failure. Am Rev Respir Dis 1979;119:547-54.

11. The Who Mers-Cov Research Group. State of Knowledge and Data Gaps of Middle East Respiratory Syndrome Coronavirus (MERS-Cov) in Humans. PLoS Curr 2013;12:5.

12. Wong KT, Antonio GE, Hui DS, Lee N, Yuen EH, Wu A, et al. Severe acute respiratory syndrome: Radiographic appearances and pattern of progression in 138 patients. Radiology 2003;228:401-6.

13. Wong KT, Antonio GE, Hui DS, Lee N, Yuen EH, Wu A, et al. Thin-section CT of severe acute respiratory syndrome: Evaluation of 73 patients exposed to or with the disease. Radiology 2003;228:395-400.

14. Hansell DM, Bankier AA, MacMahon H, McLoud TC, Müller NL, Remy J. Fleischner Society: Glossary of terms for thoracic imaging. Radiology 2008;246:697-722.

15. Ooi GC, Daqing M. SARS: Radiological features. Respirology 2003;8:S15-9.

16. Austin JH, Müller NL, Friedman PJ, Hansell DM, Naidich DP, 
Remy-Jardin M, et-al. Glossary of terms for CT of the lungs: Recommendations of the Nomenclature Committee of the Fleischner Society. Radiology 1996;200:327-31.

17. Collins J, Stern EJ. Chest radiology: The essentials. Philadelphia: Wolters Kluwer Health/Lippincott Williams \& Wilkins; 2008. p. 34-47.

18. Copley S, Hansell DM, Kanne JP. Thoracic imaging, CRC Press 2014:158-9.

19. Muller NL, Silva CIS. Mediastinum. In: Muller NL, Silva CIS, editors. Imaging of the chest. Philadelphia, Pa: Elsevier Saunders: 2008. p. 1447-572.

20. Lee N, Hui D, Wu A, Chan P, Cameron P, Joynt GM, et al. A major outbreak of severe acute respiratory syndrome in Hong Kong. N Engl J Med 2003;348:1986-94.

21. Chiang CH, Shih JF, Su WJ, Perng RP. Eight-month prospective study of 14 patients with hospital-acquired severe acute respiratory syndrome. Mayo Clin Proc 2004;79:1372-79.

22. Ooi GC, Khong PL, Müller NL, Yiu WC, Zhou LJ, Ho JC, et al. Severe acute respiratory syndrome: Temporal lung changes at thin-section CT in 30 patients. Radiology 2004;230:836-44.
23. Razer RS, Muller NL, Colman N, Pare PD. Viruses, mycoplasmas, chlamydiae, and rickettsiae. In: Fraser and Pare's diagnosis of diseases of the chest. $4^{\text {th }}$ ed. Philadelphia, PA: Saunders: 1999. p. $979-1032$.

24. Mineo G, Ciccarese F, Modolon C, Landini MP, Valentino M, Zompatori M. Post-ARDS lung fibrosis in patients with H1N1 pneumonia: Role of follow-up CT. Radiol Med 2012;117:185-200.

25. Antonio GE, Wong KT, Hui DS, Wu A, Lee N, Yuen EH, et al. Thin-section $\mathrm{CT}$ in patients with severe acute respiratory syndrome following hospital discharge: Preliminary experience. Radiology 2003;228:810-5.

26. Naik PK, Moore BB. Viral infection and aging as cofactors for the development of lung fibrosis. Expert Rev Respir Med 2010;4:759-71.

27. Moore BB, Moore TA. Viruses in Idiopathic Pulmonary Fibrosis. Etiology and Exacerbation. Ann Am Thorac Soc 2015;12:S186-92.

28. Molyneaux PL, Cox MJ, Willis-Owen SA, Mallia A, Russell KE, Russell AM, et al. The role of bacteria in the pathogenesis and progression of idiopathic pulmonary fibrosis. Am J Respir Crit Care Med 2014;190:906-13. 